\title{
3D Reconstruction Based on Fusing Active Structured Laser and Passive Stereo Techniques
}

\author{
Shahad A. Al-Saqal ${ }^{1}$, Ali A. Al-Temeemy ${ }^{2 *}$
}

\section{Authors affiliations: \\ 1) Laser and Optoelectronics Eng. Dept., Al-Nahrain University, Baghdad-Iraq. shahadahmed.94@gmail.com}

$\left.2^{*}\right)$ Laser and Optoelectronics Eng. Dept., Al-Nahrain University, Baghdad-Iraq. ali.al temeemy@eng.nahrainun iv.edu.iq

\section{Paper History:}

Received: $13^{\text {th }}$ Feb. 2020

Revised: $24^{\text {th }}$ April 2020

Accepted: $14^{\text {th }}$ Aug. 2020

\begin{abstract}
Three-dimensional reconstruction of real objects comprises capturing the appearance and the shape for these objects and determining the three-dimensional coordinates for their profiles. This reconstruction process can be accomplished either by using active or passive techniques. In this paper, a new fusion method is proposed for $3 \mathrm{D}$ reconstruction. This method exploits the advantages of both stereo-based passive and laser-based active techniques and overcomes their limitations to improve the performance of $3 \mathrm{D}$ reconstruction. With this method, a hybrid laserbased structured light scanning system is designed and implemented. This system captures the required information using passive and active techniques and uses the proposed fusion method for $3 \mathrm{D}$ reconstruction. The performance of the proposed method and its scanning system were experimentally evaluated. The evaluation results show high reconstruction performance for the proposed fusion method over the traditional 3D reconstruction techniques. The results also show the effectiveness of the hybrid laser scanning system and its ability to scan and reconstruct the shape and the appearance for real objects using the proposed fusion method.
\end{abstract}

Keywords: 3D Reconstruction, Multi-view Vision, Stereo Vision, Structured Laser.

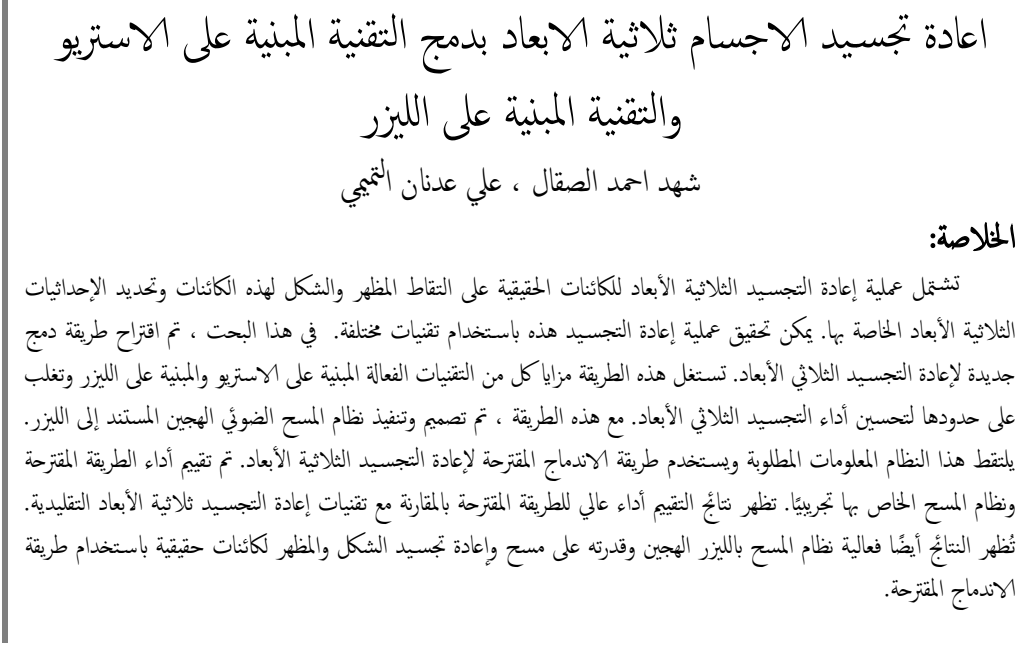

\section{Introduction}

Three dimensional (3D) reconstruction's researches have been increased in the recent years in many different fields such as computer vision, medicine, multi-media, virtual reality, and many other fields [1, 2]. Different techniques have been proposed for reconstructing the $3 \mathrm{D}$ information for the scanned objects. These techniques can be categorized into active and passive techniques [3, 4].

Stereo vision is one of the passive 3D reconstruction techniques that computes the $3 \mathrm{D}$ structure of a scene from two dimensional (2D) images. It usually uses two cameras to estimate the 3D structure by using the principle of triangulation $[5,6]$.

The researchers used this technique in different applications. Chae and Kano [7], presented an application of stereo vision in location information to monitor project progress in actual construction site. Chiang et al. [8], used stereo vision for robotics' applications. They developed a 3D position stereo vision system able to measure the $3 \mathrm{D}$ trajectories of the robotic arm. In the same field, Grigorescu et al. [9], proposed a stereo vision system able to detect the objects' structure located in the robotic scene and also

NJES is an open access Journal with ISSN 2521-9154 and eISSN 2521-9162

This work is licensed under a Creative Commons Attribution-NonCommercial 4.0 International License 
able to estimate the relative position between the robot and the detected objects.

Stereo vision technique reconstructs the $3 \mathrm{D}$ information of real objects by finding the corresponding points on theirs surfaces. This vital process is mainly based on the surfaces' texture for these objects. This limits the reconstruction ability for the stereo vision technique and makes it unable to deal with texture-less or free-form objects $[10,11]$.

In active techniques, the researchers solved the corresponding problem through creating an artificial texture on the texture-less surfaces using structured laser pattern. $\mathrm{Li}$ et al. [12], presented a 3D reconstruction system, consists from a single laser line projector, turntable, and monocular camera for indoor application. He et al. [13], used a cross structured light projector emits two mutually perpendicular laser beams with colour camera to perform the 3D reconstruction. Jia et al. [14], designed a grid pattern generated by laser diffraction with a single camera to measure the object depth of the surrounding scene in real time.

Active techniques based on using structured laser with a single camera are able to deal with texture-less surfaces, but their systems required laser scanning stage with high precision components. In addition two calibration procedures must be performed for these systems, one for the laser plane and the other for camera's parameters estimation [15].

To overcome these limitations, the researchers used two cameras with structure laser instead of a single camera. Davis and Chen [16], designed a system able to estimate the depth by using hand-held laser with two virtual cameras (like stereo vision) made by combining one camera with two mirrors. Unlike the previous system, Li et al., [17] presented a low-cost desktop 3D laser scanner consist of a hand-held laser line and two webcams for image acquisition instead of virtual cameras. Liu et al. [18], presented stereo vision system with laser dot matrix projector for $3 \mathrm{D}$ measurements. This projector used two orthogonal fine grating with laser diode to generate dot matrix with high contrast. Xiong et al. [19], also used multi views principle with structured laser. But instead of strip or dot matrix, they projected grid of laser lines aiming to reconstruct the free-form surface which has uncertain texture and uncertain shape.

In addition to the previously mentioned techniques, the researchers improved the $3 \mathrm{D}$ reconstruction's performance even further by combining both passive and active techniques. Guðmundsson et al. [20], presented an approach of using passive stereo vision system with SwissRanger Time-Of-Flight (TOF) active system to improve the 3D estimation. Kim et al. [21, 22], used the same principle but they combined the information from multiple passive active TOF systems. Peng et al. [23, 24], used 3D laser radar (based on single-pulse timeof-flight) and stereo-vision systems for high-precision depth estimation, pose measurements, and motion estimation. Beside TOF depth sensors, Yilmaz and Karakus [25] introduced the principle of using IR random-pattern depth system with stereo system for visual odometry applications. As previously mentioned, the principle of combining passive and active vision systems improves the reconstruction performance, but leads to increase the cost, size and usage power. For these reasons, Yemez and Wetherilt [26], estimated the 3D information from a single sensors' setup, using passive-shape from silhouette technique and laser scanning active-technique. Pacheco et al. [27], used the same techniques in [26] but, with high resolution camera and different Laser projector. Maurer et al. [28], presented a compact 3D system, through the use of passive-stereo vision and active-shape from shading techniques. Bylow et al. [29], also used photometric stereo and shape from shading technique but with different illumination's conditions.

Using shape from silhouette as a passive technique produces a compact system' setup, but its unable to deal with concave-regions and it's difficult to implement it in real conditions [30]. In contrast, using shape from shading as an active technique also minimizes the setup's requirements, but this technique is mathematically unstable and depends on the illumination and brightness of the scene [31].

To address these limitations, a cost effective laserbased structured light scanning system is designed and implemented. This system based on proposed method that combines the advantages of both stereo-based passive and laser-based active techniques to improve the performance of $3 \mathrm{D}$ reconstruction.

In the following sections, an overview about the $3 \mathrm{D}$ reconstruction methodology for passive and active techniques is given. A description follows of the proposed fusion method and how it used to overcome the limitations associated with traditional techniques. The experimental hardware setup and the required calibration procedure are described. This is followed by experimental procedure, results, discussion and conclusion.

\section{3D Reconstruction Methodology}

The methodology of reconstruction the depth information from multi-views vision system with using laser illumination (active technique) or without using it (passive technique), involves applying different processing stages on the images of that system. These stages include: performing stereo calibration, defining epipolar geometry, applying rectification, finding the corresponding points, and finally determining the disparity and estimating the depth from it.

\subsection{Stereo calibration}

Stereo calibration is the process of determining the required parameters for multi-views vision system with two cameras. These parameters are essential for 3D reconstruction and they categorized into intrinsic, extrinsic, and distortion parameters.

\subsubsection{Intrinsic parameters}

In vision system, the intrinsic parameters are the parameters that describe the manufacturer's specification for each camera in multi-views vision system. These parameters are the focal length of the lens, principle point, size and the aspect ratio of the image sensor pixels. They used to describe the central projection model responsible for projecting $3 \mathrm{D}$ world into the camera image plane. 
In this model the image plane contains a (Width $\boldsymbol{W} \times$ Height $\boldsymbol{H}$ ) grid of pixels; their coordinates $(\mathrm{u}, \mathrm{v})$ are nonnegative integers with origin located at the top-left as shown in Fig. 1 [32].

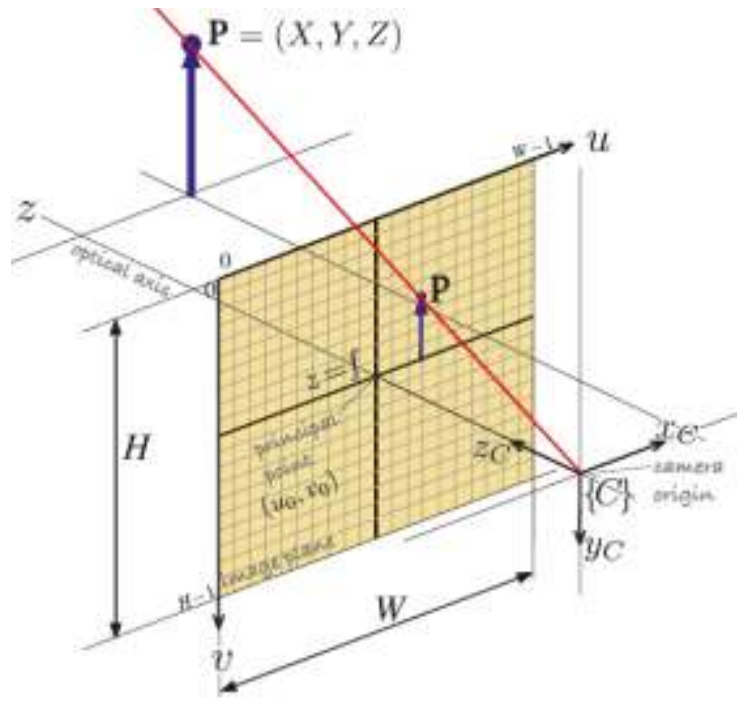

Figure (1): Central projection model for discrite image plane [32]

Referring to the Fig. 1, the camera matrix for the central projection model can be written as [32] $\widetilde{\mathbf{p}}(\tilde{\mathrm{u}}, \tilde{\mathrm{v}}, \widetilde{\mathrm{w}})=$

$\underbrace{\left(\begin{array}{ccc}1 / \rho_{\mathrm{w}} & 0 & \mathrm{u}_{0} \\ 0 & 1 / \rho_{\mathrm{h}} & \mathrm{v}_{0} \\ 0 & 0 & 1\end{array}\right)}_{\kappa}\left(\begin{array}{cccc}\mathrm{f} & 0 & 0 & 0 \\ 0 & \mathrm{f} & 0 & 0 \\ 0 & 0 & 1 & 0\end{array}\right){ }^{\mathrm{C}} \widetilde{\mathbf{P}}$

where $\tilde{\mathrm{p}}=(\tilde{\mathrm{u}}, \tilde{v}, \widetilde{\mathrm{w}})$ and ${ }^{\mathrm{C}} \widetilde{\mathrm{P}}=(\mathrm{X}, \mathrm{Y}, \mathrm{Z}, 1)^{\mathrm{T}}$ are the homogenous coordinates for world point $\mathrm{P}$ in pixel and world (with respect to the camera frame $\{C\}$ ) coordinates respectively, $\mathrm{u}=\tilde{\mathrm{u}} / \widetilde{\mathrm{w}}$ and $\mathrm{v}=\tilde{\mathrm{v}} / \widetilde{\mathrm{w}}$ are non-homogenous pixel coordinates, $f$ is the focal length, and $\kappa$ is the camera parameter matrix. This matrix contains the principle point $\left(\mathrm{u}_{0}, \mathrm{v}_{0}\right)$ and pixel size defined by $\rho_{\mathrm{w}}$ and $\rho_{\mathrm{h}}$ which represent the width and height of each pixel respectively [32].

\subsubsection{Extrinsic parameters}

It has been shown that with the intrinsic parameters, the relation between the point at world coordinate and its projection at image plane was defined with respect to the camera frame. Generally the image's projection is calculated with respect to the world frame $\{0\}$ as shown in Fig. 2 [32]. Therefore the extrinsic parameters are required, which they represent the position and the orientation of the camera relative to the scene [33].

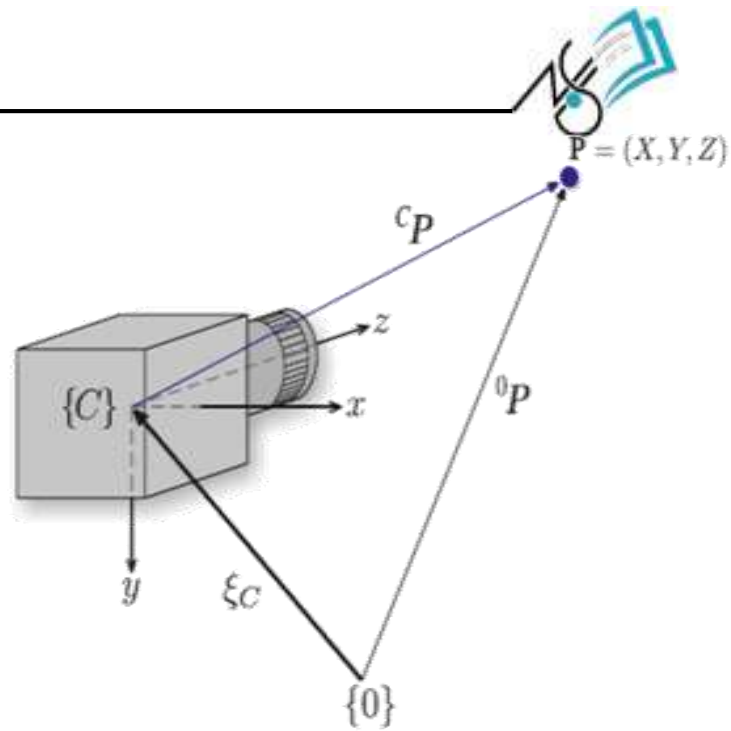

Figure (2): Camera coordinate frames [32]

Referring to the Fig. 2, ${ }^{\mathbf{C}} \mathbf{P}$ and ${ }^{\mathbf{0}} \mathbf{P}$ are the world coordinates for point $\mathrm{P}$ with respect to the camera frame $\{C\}$ and world frame $\{0\}$ respectively. $\xi \mathrm{c}$ is the camera's position with respect to the world coordinate frame $\{0\}$. With adding the extrinsic parameters, the camera matrix becomes [32]:

$\tilde{\mathrm{p}}(\tilde{\mathrm{u}}, \tilde{\mathrm{v}}, \widetilde{\mathrm{w}})=$

$\underbrace{\left(\begin{array}{ccc}\mathrm{f} / \rho_{\mathrm{w}} & 0 & \mathrm{u}_{0} \\ 0 & \mathrm{f} / \rho_{\mathrm{h}} & \mathrm{v}_{0} \\ 0 & 0 & 1\end{array}\right)\left(\begin{array}{llll}1 & 0 & 0 & 0 \\ 0 & 1 & 0 & 0 \\ 0 & 0 & 1 & 0\end{array}\right)} \underbrace{\left({ }^{0} \mathrm{~T}_{\mathrm{C}}\right)^{-1}}_{\text {extrinsic }} \widetilde{\mathrm{P}}$

Stereo calibration process not only estimates the camera's position with respect to the world coordinate frame but also estimates the relative position between the multi-views system's cameras [33].

\subsubsection{Distortion parameters}

Typical digital cameras have distortions due to lens imperfection [33]. Among different lens-distortions' types, the most problematic effect in 3D reconstruction comes from the radial and tangential distortions [32]. The first results from the lens' shape, while the other occurs when the sensor is not parallel to the vertical plane $[34,32]$. If the parameters for these distortions' type are estimated, their effects can be removed from the projected point $[35,36]$.

The point coordinate with distortion is given by [32]

$\mathrm{u}^{\mathrm{d}}=\mathrm{u}+\delta_{\mathrm{u}}, \quad \mathrm{v}^{\mathrm{d}}=\mathrm{v}+\delta_{\mathrm{v}}$

$$
\begin{aligned}
& \left(\begin{array}{c}
\delta_{\mathrm{u}} \\
\delta_{\mathrm{v}}
\end{array}\right)=\underbrace{\left(\begin{array}{l}
\mathrm{u}\left(\mathrm{k}_{1} \mathrm{r}^{2}+\mathrm{k}_{2} \mathrm{r}^{4}+\mathrm{k}_{3} \mathrm{r}^{6}+\cdots\right) \\
\mathrm{v}\left(\mathrm{k}_{1} \mathrm{r}^{2}+\mathrm{k}_{2} \mathrm{r}^{4}+\mathrm{k}_{3} \mathrm{r}^{6}+\cdots\right)
\end{array}\right)}_{\text {radial }}+ \\
& \underbrace{\left(\begin{array}{l}
2 \mathrm{p}_{1} \mathrm{uv}+\mathrm{p}_{2}\left(\mathrm{r}^{2}+2 \mathrm{u}^{2}\right) \\
\mathrm{p}_{1}\left(\mathrm{r}^{2}+2 \mathrm{v}^{2}\right)+2 \mathrm{p}_{2} \mathrm{uv}
\end{array}\right)}_{\text {tangential }}
\end{aligned}
$$

Where $\mathbf{k}_{\mathbf{1}, 23}$ and $\mathbf{p}_{\mathbf{1}, \mathbf{2}}$ are the radial and tangential distortion coefficients respectively, and $r$ is the distance of the image point to the principle point [32].

\subsection{Epipolar geometry}

After applying stereo calibration and determining the required system's parameters including the relative position between the system's cameras, the Epipolar geometry is then used to simplify the searching process. Figure 3 illustrates this geometry and shows the system's two cameras with their coordinates frames $\{1\}$ and $\{2\}$ [32]. 


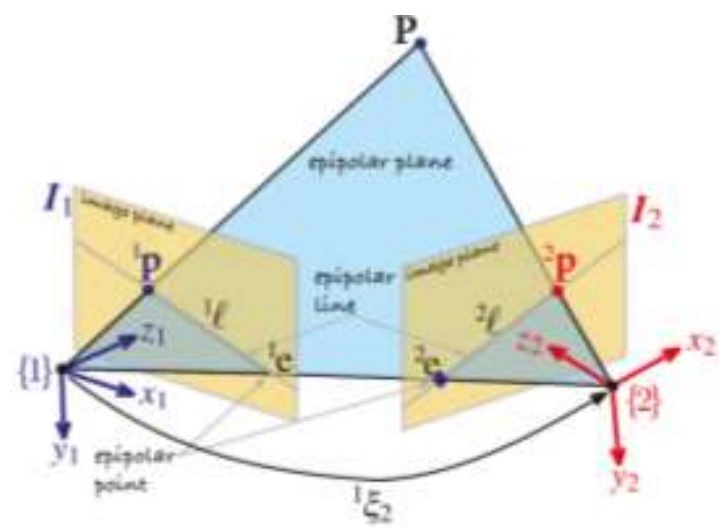

Figure (3): The Epipolar geometry [32]

Since the 3D reconstruction is based on the triangulation principle. The position of the world point $\mathrm{P}$ can by calculated from its projections ${ }^{1} \mathrm{p}$ and ${ }^{2} \mathrm{p}$ on the image planes $I_{1}$ and $I_{2}$ respectively [37].

Referring to Fig. 3, the image point ${ }^{1} \mathrm{p}$ is a function of the world point $\mathrm{P}$. The camera center, ${ }^{1} \mathrm{e}$ and ${ }^{1} \mathrm{p}$ define the epipolar plane and hence the epipolar line ${ }^{2} l$ in image plane two $\mathrm{I}_{2}$. By definition the point ${ }^{2} \mathrm{p}$ must lie on that line $2 \ell$. Therefore this geometry is used to simplify the search through identifying which line ${ }^{2} l$ at image $\{2\}$ corresponds to the point ${ }^{1} \mathrm{p}$ at image $\{1\}$. This line is defined by [32]

$$
\begin{gathered}
{ }^{2} \tilde{\ell} \simeq \mathrm{F}^{1} \tilde{\mathrm{p}} \\
\mathrm{F} \simeq \mathrm{K}_{2}^{-\mathrm{T}}[\mathrm{t}]_{\mathrm{X}} R \mathrm{~K}_{1}^{-1}
\end{gathered}
$$

where ${ }^{1} \tilde{p}$ is the homogeneous image points, $\mathrm{F} \subset \mathrm{R}^{3 \times 3}$ is the fundamental matrix, $K_{1}, K_{2}$ are the camera matrices parameters defined in equation (1), and $\mathrm{R}, \mathrm{t}$ are the relative position of camera one with respect to camera two.

\subsection{Rectification}

The searching process can be simplified even further in the epipolar lines through applying rectification on the system's pair images. This process makes the corresponding epipolar lines in two image planes become collinear with each other and the image scanning lines (image rows) perfectly aligned into a frontal parallel configuration [38].

Figure 4 shows the process of rectification on the input pair images shown in Fig. 4(a). The figure shows how the distortion is removed at the beginning of the process (Fig. 4(b)), it then shows how the rectification aligns the epipolar lines for the undistorted images (Fig. 4(c)). After rectification not all images' parts are helpful for reconstructing depth information. Therefore, the helpful part of the rectified images is cropped as shown in Fig. 4(d) [39]. (a)

Left camera

Object

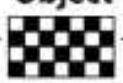

Right camera

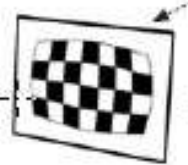
Raw images

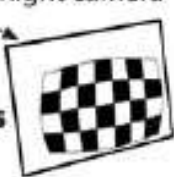

(b)

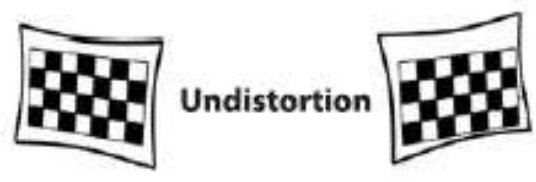

(c)

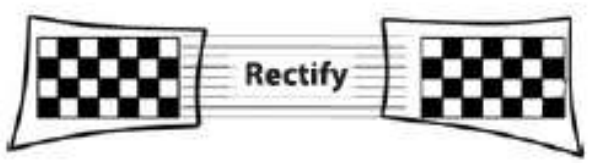

(d)

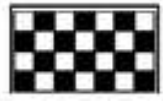

Crop

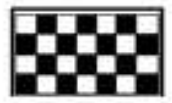

Figure (4): The image rectification [39].

\subsection{Corresponding points \& Disparity}

Corresponding points are the projections of the same point $\mathrm{P}$ on the object's surface in two image planes [40]. These points are used with the triangulation principle to reconstruct the $3 \mathrm{D}$ coordinate for that point $\mathrm{P}$, therefore finding them is fundamental in 3D reconstruction methodology. Corresponding points identification can be implemented by making the searching process in a single dimension through rectification and then applying feature matching or laser-line segmentation depending on the reconstruction techniques ( passive or active) [39].

\subsubsection{Feature matching}

For passive technique the search for corresponding points acquire by feature matching process. Feature matching is the process of finding which pixel in the left rectified image has the most feature-similarity with a specific pixel in the right rectified image [41, 42].

Figure 5 shows how the feature matching searches for the corresponding points $\left({ }^{1} \mathrm{p},{ }^{2} \mathrm{p}\right)$ in the rectified stereo pair. It shows the similarity values (white peaks at the bottom) across the searching range. It also shows the disparity $(d)$ for corresponding points and their distances in the left $\left(D_{L}\right)$ and the right $\left(D_{R}\right)$ images [32].

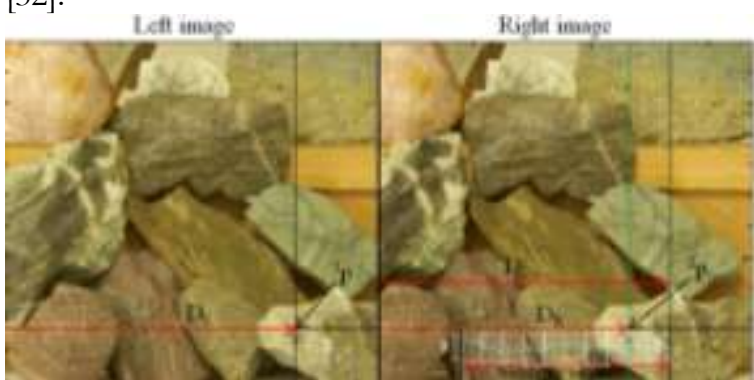

Figure (5): The search of a correspondent pixel in the rectified stereo pair [32].

\subsubsection{Laser line segmentation method}

For active technique the corresponding points' identification can be implemented by applying laser 
line segmentation method on the rectified stereo pair. This method extracts the laser line from the rectified images and determines the center location for this line at each row [43].

Figure 6 shows how the extracted laser' center locations that represent the corresponding points $\left({ }^{1} \mathrm{p}\right.$, $\left.{ }^{2} \mathrm{p}\right)$ at specific row in the rectified stereo pair. It also shows the disparity $(d)$ for corresponding points' and their distances in the left $\left(D_{L}\right)$ and the right $\left(D_{R}\right)$ images.

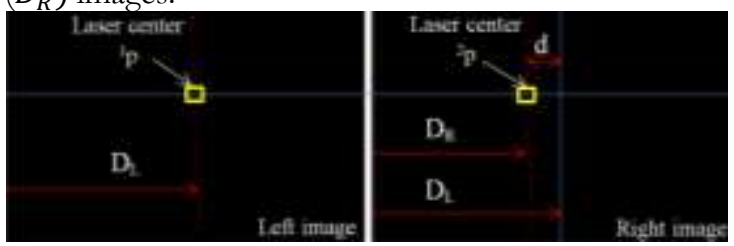

Figure (6): Disparity calculation for the laser line in a rectified stereo pair at a specific row.

\subsection{Depth estimation}

Estimating the depth is the final stage in the 3D reconstruction. It uses the calculated correspondingpoints' disparities from the stereo pairs with the principle of triangulation for depth calculation [44, 45].

Figure 7 shows a top-view of two perfectly aligned cameras (using rectification process) of origins $\{1\}$ (left), $\{2\}$ (right) and focal length $f$, separated from each other by a distance $B$ (baseline). The projections of point $P$ on the left and right image-planes (pinklines), are represented by ${ }^{1} \mathrm{p}$ and ${ }^{2} \mathrm{p}$ respectively [39].

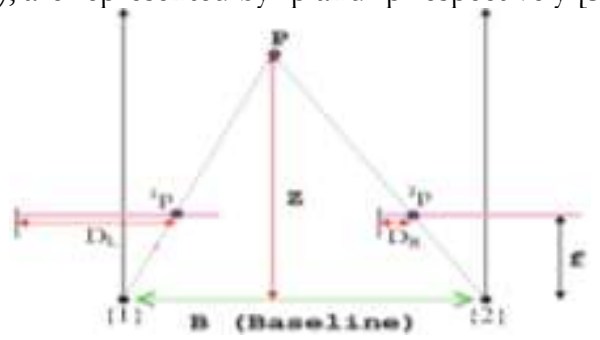

Figure (7): Depth estimation using two rectified cameras [39]

Referring to the above figure, the depth of a $3 \mathrm{D}$ point $P$ can be obtained using following relation [39]:

$$
\mathrm{Z}=\frac{\mathrm{Bf}}{\mathrm{D}_{\mathrm{L}}-\mathrm{D}_{\mathrm{R}}}
$$

where $D_{L}-D_{R}$ is the disparity and $Z$ is the depth of point $\mathrm{P}$ [39].

\section{Proposed Fusion Method}

The proposed fusion method improves the 3D reconstruction through combining stereo-based passive and laser-based active techniques. It exploits the high resolution at fast acquisition speed for the stereo passive technique using feature matching process with the high accuracy and feature-less robustness for the active structured-laser-light technique. Unlike the other fusion methods that combined different sensing systems, the proposed fusion method reduces the cost, size and usage power through the use of a single setup's sensing-elements for both passive and active acquisitions. In addition this method didn't used shape from silhouette and shape from shading techniques that previously proposed with a single-setup's system because of the previously mentioned limitations.
The steps required for implementing the proposed fusion method are shown in the flowchart of Fig. 8. These steps are:

- Disparity maps calculation from the input recorded frames using stereo-based passive and Laser-based active techniques.

- Since the disparity map result from active techniques is robust to the feature-less objects, its values are used to calculate the percentage of difference with the map of passive technique. This is by applying average filter on the active map to replace the missing values with approximate depth values and then dividing the absolute difference between this processed active-map and the passive- map, by its values.

- Based on a specific percentage threshold. A binary mask is created by applying hard-thresholding process on the resultant map of percentagedifference.

- The resultant mask is applied on the passive disparity map to filter-out the invalid disparity values.

- The fusion process is then performed by filling the gaps in the active disparity map with the reliable values in the filtered passive disparity map.

- The 3D information is then reconstructed using the resultant filled active-disparity map and other calibration parameters.

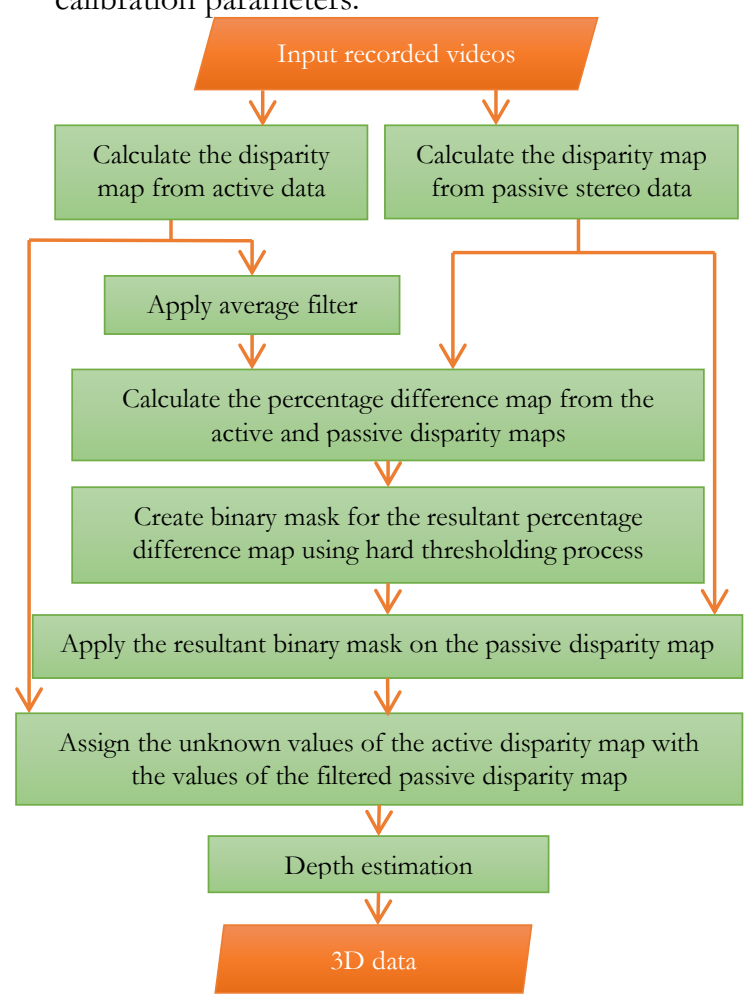

Figure (8): Steps of the proposed fusion method.

\section{Structured Laser Scanning System}

A cost effective hybrid laser-based structured light scanning system has been developed to scan real objects and reconstructs their 3D information using the proposed fusion method. This system captures the required information for this method using stereobased passive and laser-based active techniques. The 
system hardware consists of a sensing unit, Laser-line scanning unit, and personal computer to analyses the incoming information from the sensing unit as shown in Fig. 9.

The first unit consists of two Logitech c270 webcams, special holders (vertical and horizontal) for stereo arrangement, and tripod. The connection between this unit and the personal computer is accomplished through the USB connection. The laserline scanning unit shown in Fig. 10 comprises of structured laser-line projector, rotating stage, and Microcontroller with the required electronics.

- Structured Laser-line projector: it is used to project a structure laser line with a wavelength equal to $650 \mathrm{~nm}$. This projector uses laser diode as an illumination source, structured light element (raster lens) to convert the laser-diode's spot into line pattern, and mirror to project the structured light towards the scanned objects.

- Rotating stage: this stage contains stepper motor and special rotating gears. It sweeps the laser line across the scene by rotating the optical component for the laser projector.

- Microcontroller and other electronics: they used to provide the required electrical power and controlling the scanning process.

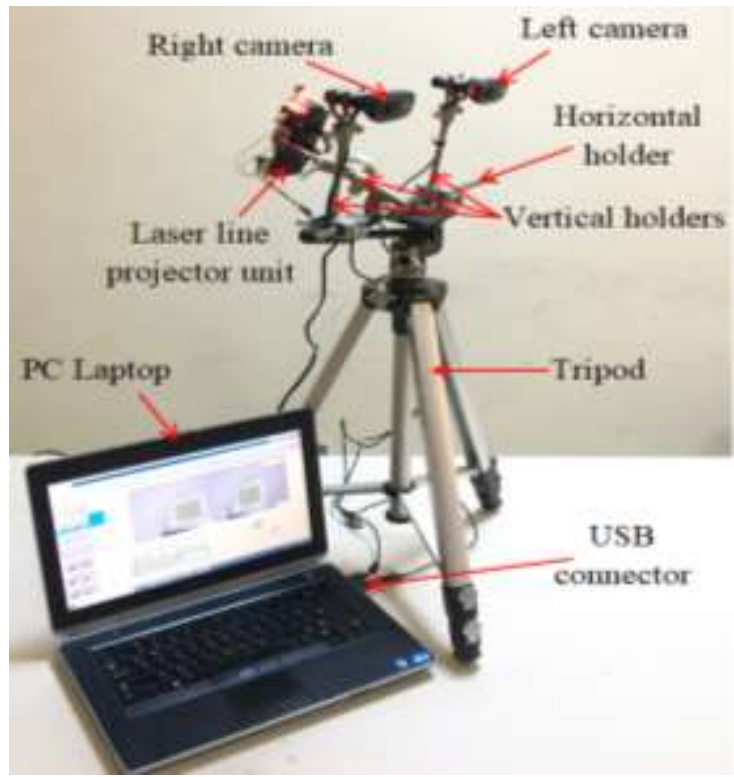

Figure (9): The system hardware.

To minimize the size, cost, and usage power, this system is designed to acquire both passive and active information from the same sensing unit. Passive information can be acquired directly from the sensing unit. Active information can be acquiring from scanning the scene with the laser line and using the sensing unit for detecting the reflected beam (Lasertwo cameras configuration).

The incoming information from the sensing unit is then processed by the personal computer, which uses the required algorithms and the designed system's parameters to reconstruct the 3D information. These parameters are the cameras intrinsic, extrinsic, lens distortion, and relative location between these two cameras.
Estimating these parameters is implemented through applying the calibration process on this system. This process includes taking images for the calibration-board at different positions as shown in Fig. 11A. This is follows by manual selection for the images that produce better estimation (minimum reprojection error Fig.11B) and ending with exporting these parameters.

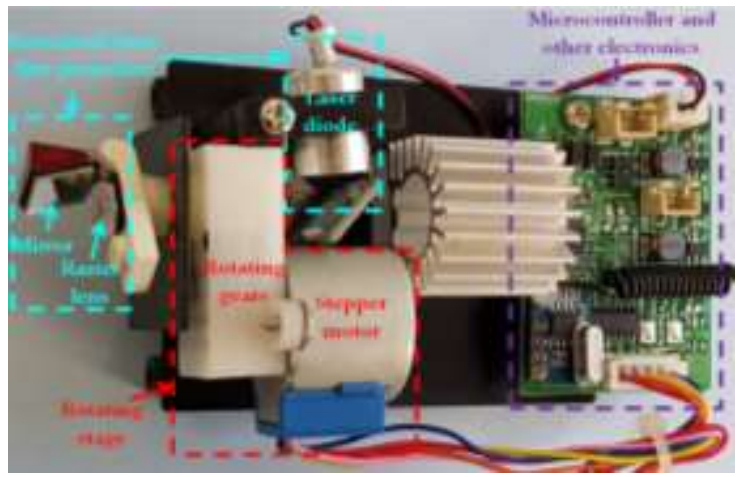

Figure (10): The laser line scanning unit

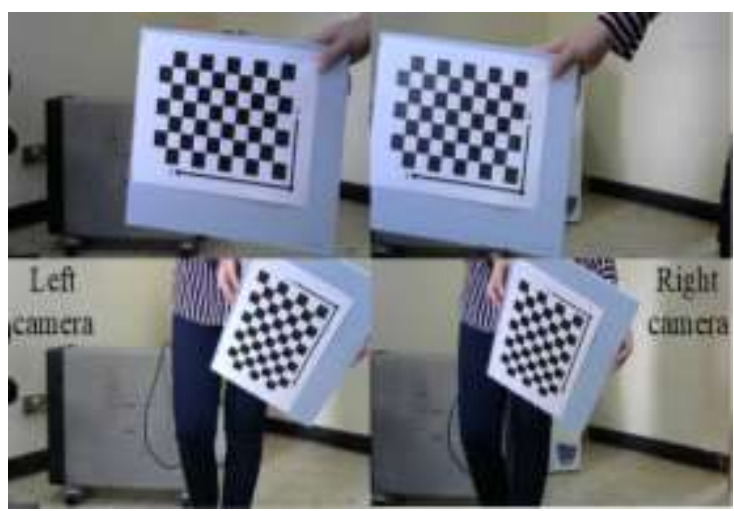

(A) Image acquisition for the calibration -board.

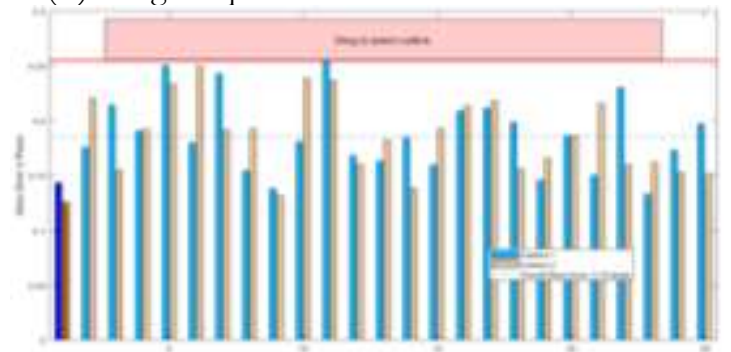

(B) Re-projection errors for each board.

Figure (11): The stereo calibration process for the hybrid Laser-scanning hardware system.

\section{Experimental Procedure and Result}

The experimental procedure of evaluating the proposed method was carried out with using laserbased structured light scanning system. This procedure consists of three stages. The first two stages are about acquiring and reconstructed the $3 \mathrm{D}$ information using passive and active techniques respectively. The third stage includes fusing the outcomes of the first two stages using the proposed fusion method.

\subsection{Passive 3D reconstruction}

The main steps of acquiring and reconstructing depth for the scanned object using passive stereo 
vision technique are presented in the flowchart of Fig. 12. With this flowchart, the experimental inputs and outputs for each step are presented in Fig. 13. These steps include:

- Acquiring free-laser images (without using laser scanning unit) using left and right cameras. Figure 13A shows the acquired cameras' frames.

- Rectifying acquired stereo pair using the rectification process to align images' lines into a frontal parallel configuration (Fig. 13B).

- Identifying the corresponding points by applying stereo matching on the rectified stereo pair.

- Calculating the disparity map from the corresponding points (Fig. 13C) and using this map with system's parameters to reconstruct the $3 \mathrm{D}$ point cloud. Figure $13 \mathrm{D}$ shows the reconstructed $3 \mathrm{D}$ point cloud for the real object shown in Fig. 13A.
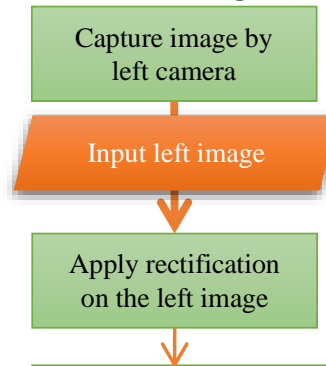
Apply feature matching between the rectified
frames (left \& right) to find the corresponding points between them

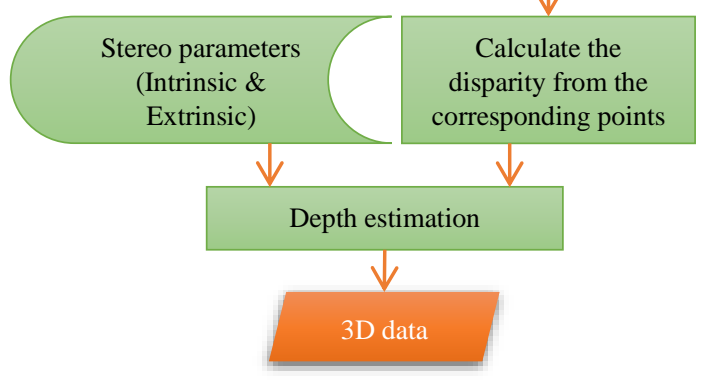

Figure (12): Steps of the passive stereo technique

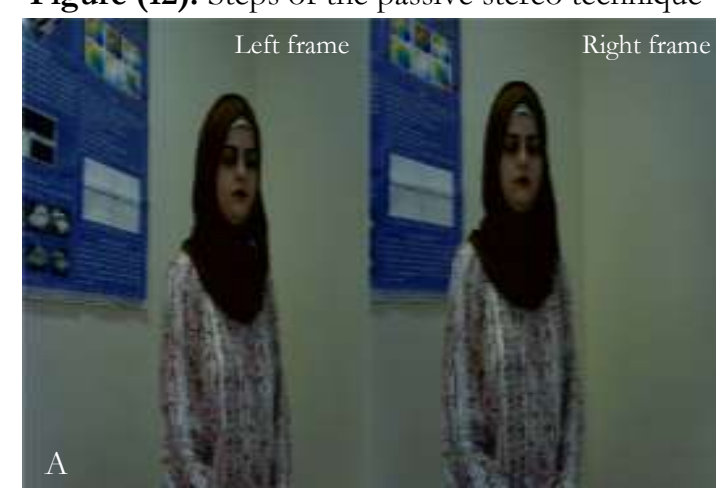

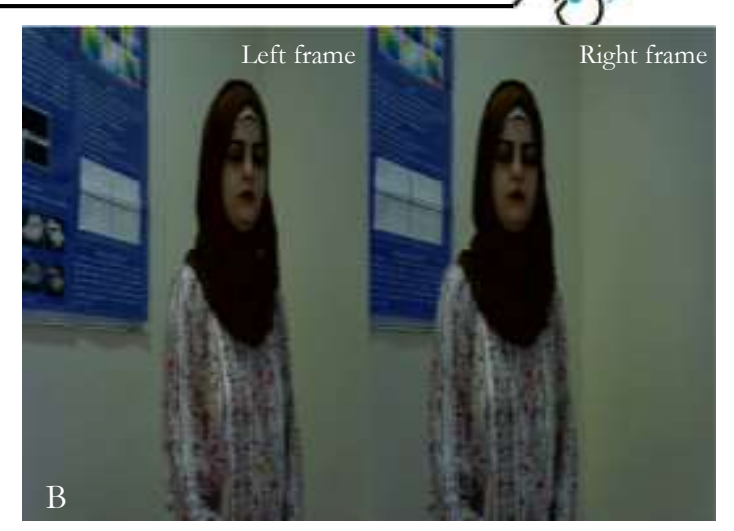
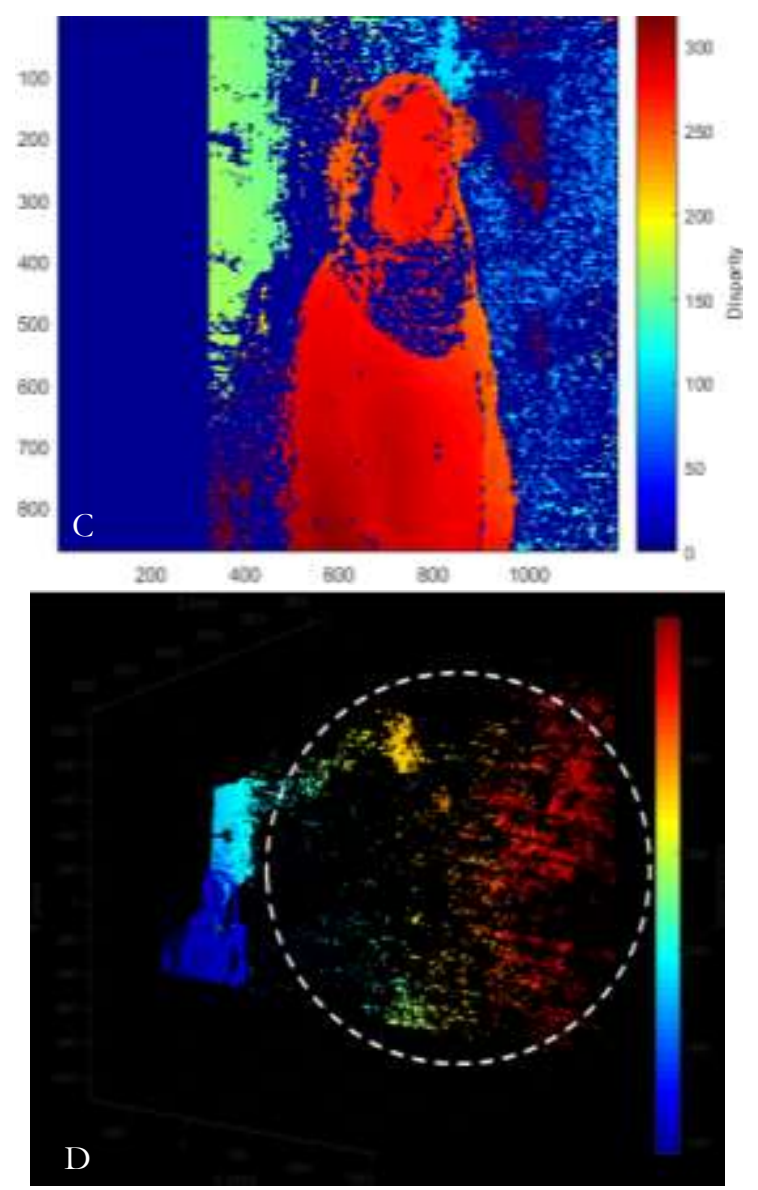

Figure (13): (A) Input and (B) rectified images, (C) disparity map, and (D) 3D point cloud (Near-Far).

\subsection{Active 3D reconstruction}

The steps of acquisition and $3 \mathrm{D}$ reconstruction using the active laser based stereo technique are presented in the flowchart of Fig. 14. In addition, the experimental inputs and outputs for each step are shown in Fig. 15. These steps include:

- Recording videos from both cameras during laser scanning process. Figure 15A shows two cameras' frames during scanning real object with structured laser line.

- Reading the recorded video files for the left and right cameras. For each individual pair frames, rectification is first applied (Fig. 15B) followed by identifying the correspondence points through extracting the laser line. The disparity is then calculated from these points and stored in the disparity map. Figure 15C shows the final disparity 
map after processing the whole frames in the video files.

- Estimating the depth from the resultant disparity map. Figure 16D shows the 3D point cloud from the object shown in (Fig. 15A).

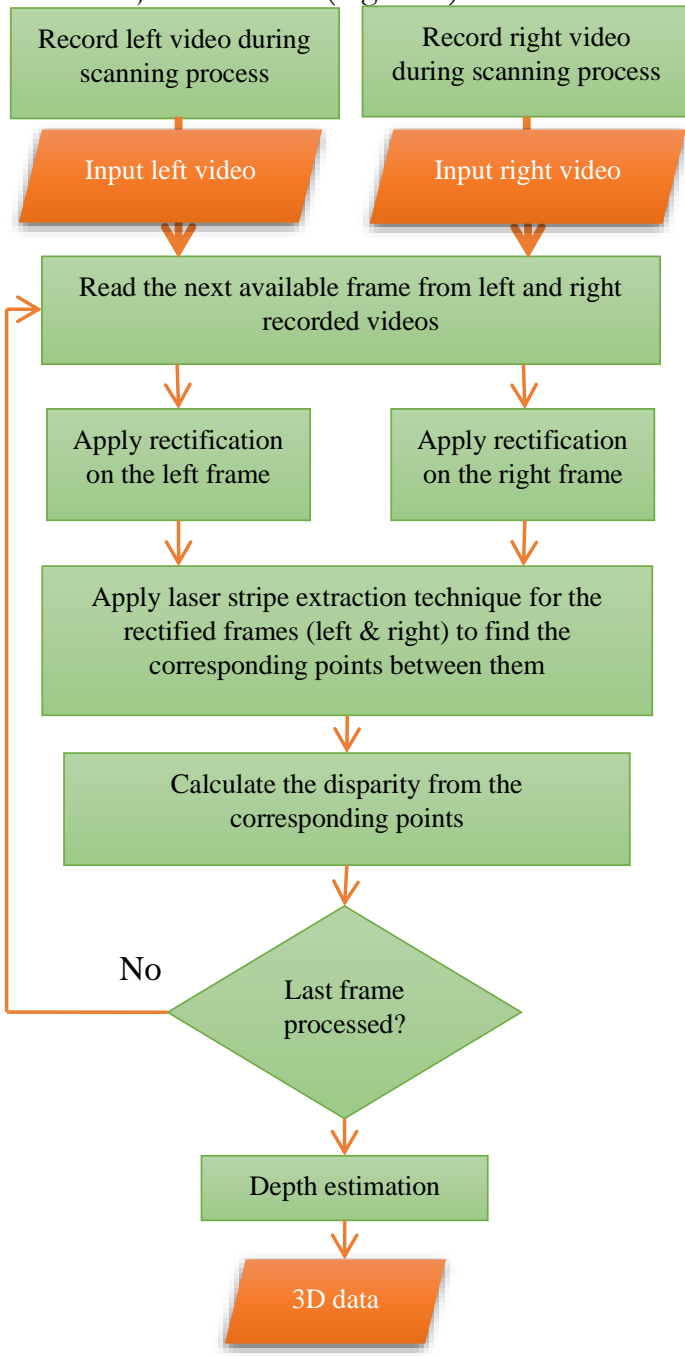

Figure (14): Steps of the active laser based stereo technique

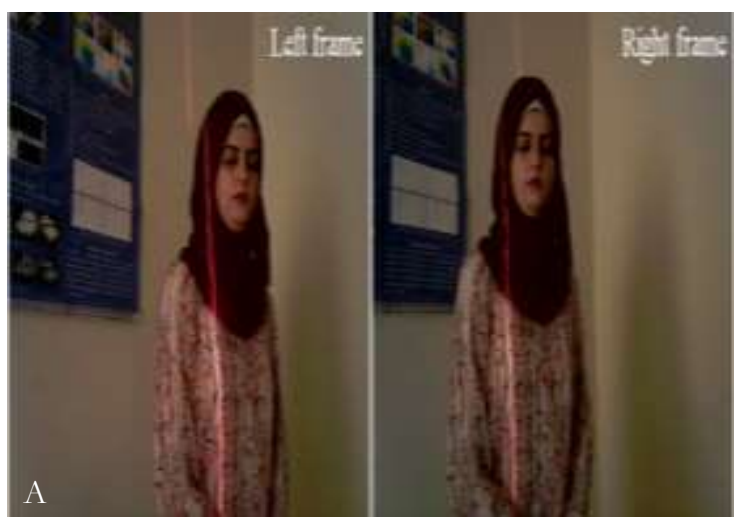

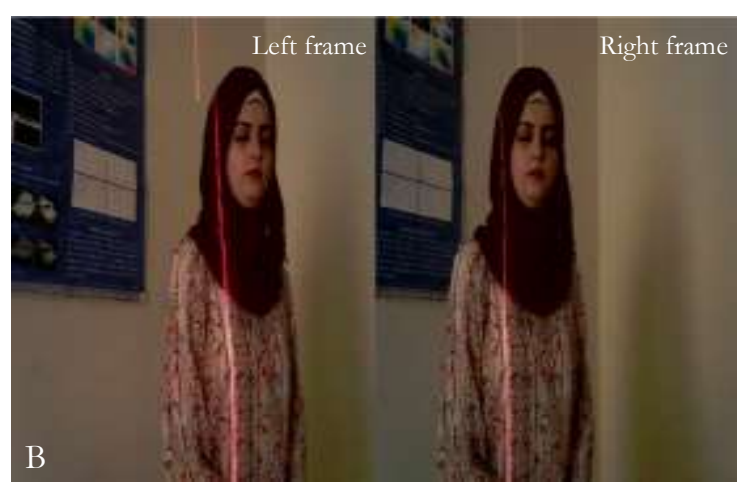

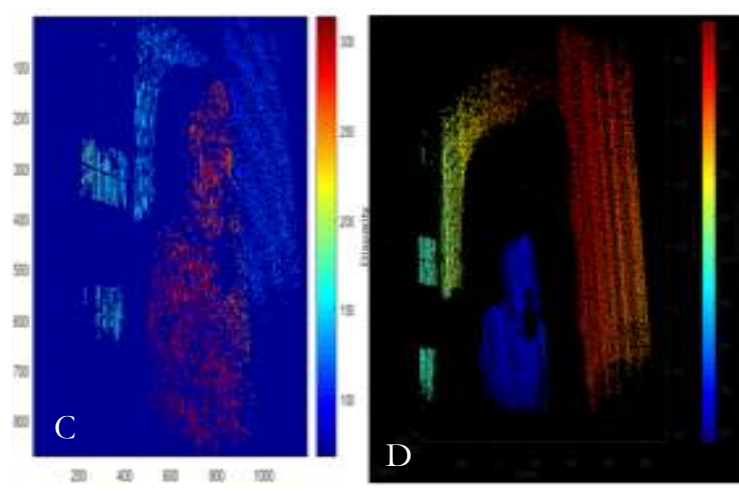

Figure (15): A) Input images. B) Rectified images. C) The disparity map. D) Resultant 3D point cloud.

\subsection{Fusing passive and active techniques}

According to the fusion-method's steps previously mentioned in section 3. The calculated passive (Fig.13C) and active (Fig. 15C) disparities in previous sections (5.1 and 5.2) are directly used with this method.

The experimental results for each fusion step with using these calculated disparities are shown in Fig. 16. In which, Fig. $16 \mathrm{~A}$ to $\mathrm{C}$ show respectively active disparity after applying average filter, the resultant difference map and the binary mask for this map. The result of applying this mask on the passive disparity to filter out unreliable depth values is shown in Fig. 16D. The fusion-disparity map result from filling the gaps in the active-disparity map (Fig. 15C) with the values in the filtered passive-disparity map (Fig. 16D) is shown in Fig. 16E. Finally the achieved 3D point cloud for the fusion-disparity map is shown in Fig. 16F. 

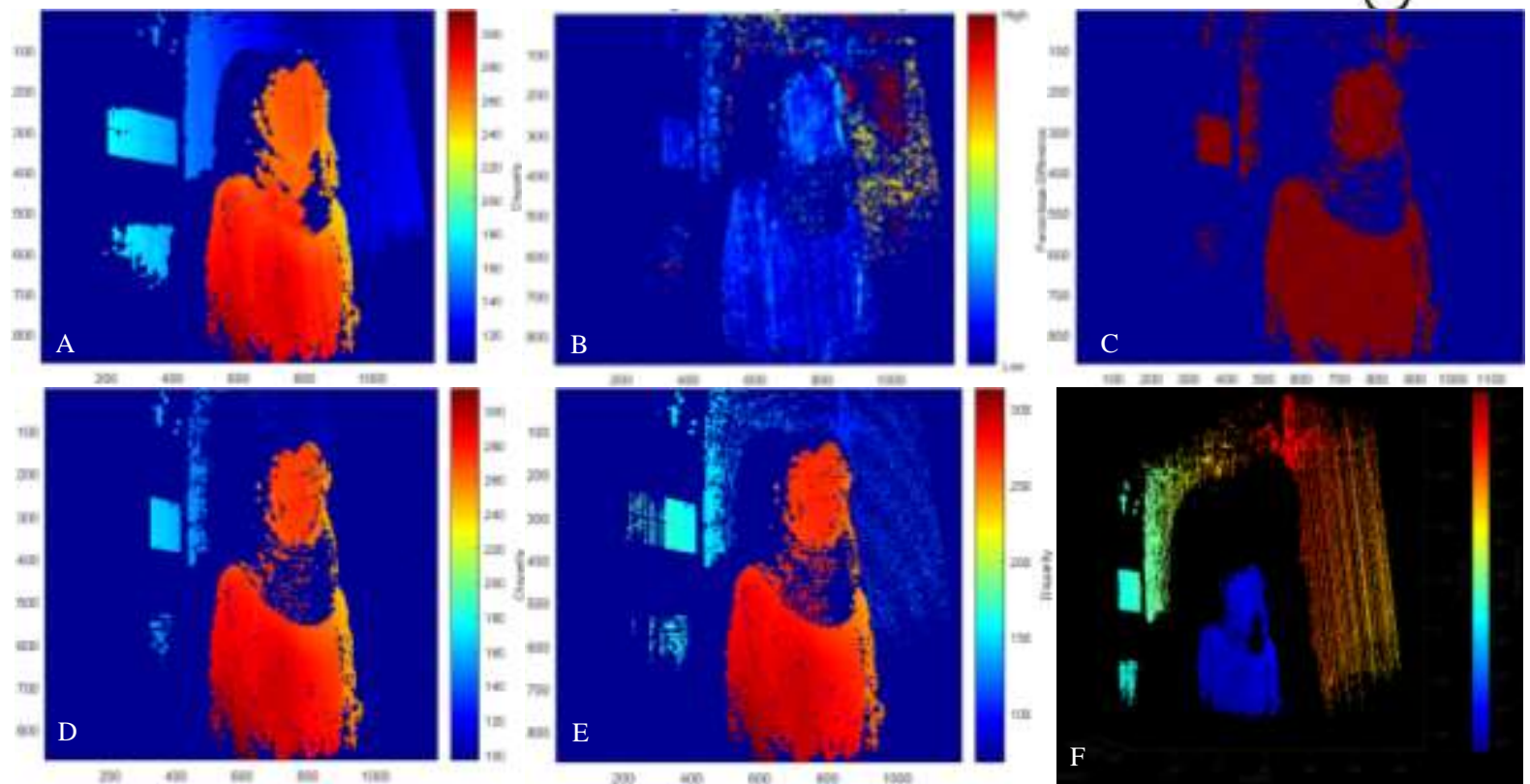

Figure (16): A) Active disparity map after average filtering, B) The percentage of difference map, C) The binary mask, D) Passive map after applying the binary mask, E) The fusion map, and F) achieved 3D point cloud.

For a comparison purpose, a close-up view for the (depth-color-coded) 3D point clouds result from using active technique and fusion method are presented side by side in Fig.17 A and B respectively. Figure $17 \mathrm{C}$ and
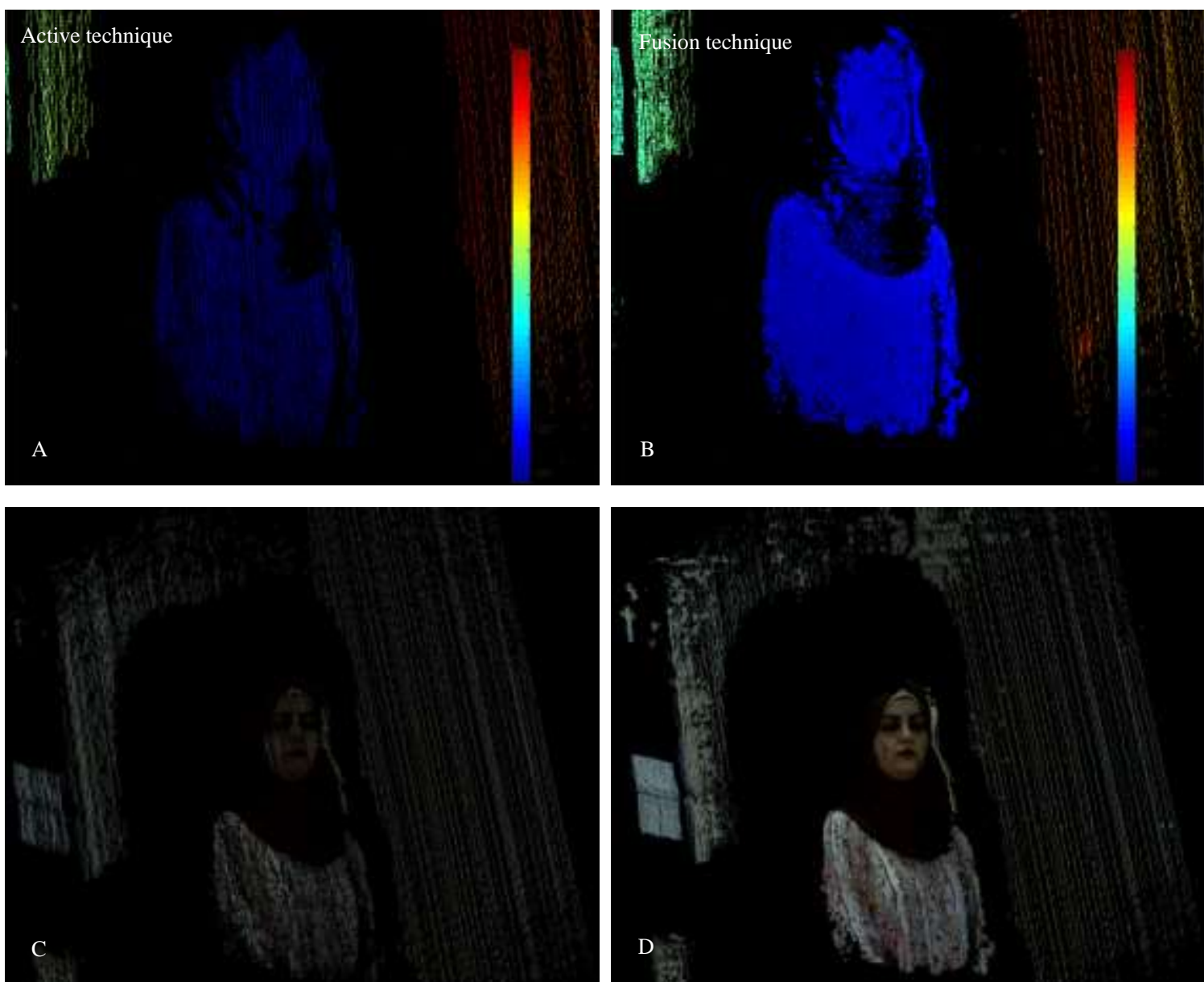

Figure (17): Close-up view of 3D point clouds for active technique (first column) and proposed fusion method (second column) presented with color coded (first row) and with true color (second row).
D show these 3D clouds after assigning the scanned object's surface color to them. 


\section{Discussion}

Regarding passive technique the experimental results show unreliable $3 \mathrm{D}$ point cloud data (white dashed circle in Fig. 13D) in some parts of the scene. These parts either have no texture (wall) or the texture's contrast in them is not enough (face region) for finding the corresponding points. With active technique, that used the laser based structured scanning unit for creating artificial texture on the scene's surface. The experimental results show reliable 3D measurement for the texture-less regions as shown in Fig. 15D, but with lower measurements number in comparison to that of the passive technique.

The experimental results for the proposed fusion method show how the unreliable measurements for the passive disparity (Fig. 13C) were filtered-out (Fig. 16D). The results also show the increment in the measurements number for the fusion disparity map (Fig.16E) in comparison to the original active disparity map (Fig. 15C). Like the previous experimental results, the resultant $3 \mathrm{D}$ point clouds for these disparities (active and fusion) presented in Fig. 17 in both color representation, also show how the missing $3 \mathrm{D}$ values are filled with filtered reliable passive 3D measurements.

\section{Conclusion}

In this paper the proposed fusion method improves the $3 \mathrm{D}$ reconstruction through combining high resolution stereo-based passive technique with the feature-less insensitive laser-based active technique.

A cost effective hybrid laser-based structured light scanning system has been developed with this proposed method. This system is able to scan real objects and captures the required information for this method using stereo-based passive and laser-based active techniques.

The experimental procedure of evaluating the designed scanning system with the proposed method was presented with the experimental results. When evaluating the hybrid laser scanning system the results show the ability for this system to acquire the required information using both passive and active techniques. The results also revealed the texture-less problem associated with passive technique and the low measurements number for the active technique. Regarding the proposed fusion method, the experimental results show the robustness of this method to extract the reliable disparity from the passive technique and using it with the active disparity to produce accurate and high resolution $3 \mathrm{D}$ reconstructed data.

\section{References:}

[1] A. A. Al-Temeemy, J. W. Spencer, "Chromatic methodology for laser detection and ranging (LADAR) image description," Optik - International Journal for Light and Electron Optics, Elsevier, Volume 126, Issue 23, Elsevier (2015), Pages 38943900, ISSN 0030-4026.

[2] A. A. Al-Temeemy and J. W. Spencer, "Laser radar invariant spatial chromatic image descriptor,"
SPIE- Opt. Eng., 53(12), 123109 (2014). doi:10.1117/1.OE.53.12.123109.

[3] S. Bai, J. Shi, Q. Ge, and Z. Tian, "Scanningbased 3D reconstruction of large-scale objects," International Conference on Robotics, Intelligent Control and Artificial Intelligence (RICAI 2019). Association for Computing Machinery, New York, NY, USA, 2019.

[4] Y. Wei, Z. Ding, H. Huang, C. Yan, J. Huang, J. Leng, "A non-contact measurement method of ship block using image-based 3D reconstruction technology," Ocean Engineering, Volume 178, Elsevier, 2019.

[5] S. Zhu, Z. Luo, Z. Zhende, Y. Gao, \& N. Wu, "Construction of Three-Dimensional Crack Calculation Method for Transparent Rock-Like Materials Based on Stereo Vision Principle," Advances in Civil Engineering, Hindawi Publishing Corporation, 2019.

[6] Y. Liu, H. Wang, C. Dong and Q. Chen, "A CarFollowing Data Collecting Method Based on Binocular Stereo Vision," in IEEE Access, vol. 8, 2020.

[7] S. Chae and N. Kano, "Application of location information by stereo camera images to project progress monitoring," Automation and Robotics in Construction - Proceedings of the 24th International Symposium on Automation and Robotics in Construction, 2007.

[8] M. H. Chiang, H. T. Lin, and C. L. Hou, "Development of a Stereo Vision Measurement System for a 3D Three-Axial Pneumatic Parallel Mechanism Robot Arm," Sensors, 2011; 11(2):22572281.

[9] S. M. Grigorescu, T. T. Cocia, G. Maceanu, and F. Moldoveanu, "Stereo vision-based 3D camera pose and object structure estimation - An Application to Service Robotics," In Proceedings of the International Conference on Computer Vision Theory and Applications - Volume 2: VISAPP, 2012.

[10] S. Barone, and A. Bruno, "Stereo Vision and Laser Stripers for Three-Dimensional Surface Measurements", XVI Congreso Internacional De Ingeniería Gráfica, 2015.

[11] M.G. Guerra, C. Volpone, L.M. Galantucci, G. Percoco, "Photogrammetric measurements of 3D printed microfluidic devices," Additive Manufacturing, Volume 21, Elsevier, 2018.

[12] D. Li, H. Zhang, Z. Song, D. Man, and M. W. Jones, "An Automatic Laser Scanning System for Accurate 3D Reconstruction of Indoor Scenes," Proceedings of the 2017 IEEE, International Conference on Information and Automation (ICIA), Macau SAR, China, July 2017.

[13] L. He, S. Wu, and C. Wu, "Robust laser stripe extraction for three-dimensional reconstruction based on a cross-structured light sensor," Applied Optics, Vol. 56, No. 4, February, 2017.

[14] T. Jia, Z. X. Zhou, and H. H. Gao, "Depth Measurement Based on Infrared Coded Structured 
Light," Journal of Sensors, Hindawi Publishing Corporation, vol. 2014, Article ID 852621, 2014.

[15] J. Li, G. Liu, and Y. Liu, "A Dynamic Volume Measurement System with Structured Light Vision", 31st Youth Academic Annual Conference of Chinese Association of Automation, IEEE, Wuhan, China, 2016.

[16] J. Davis and X. Chen, "A laser range scanner designed for minimum calibration complexity," Proceedings of Third International Conference on 3D Digital Imaging and Modeling, 2001.

[17] D. Li, B. Hui, S. Qiu, and G. Wen, “3D reconstruction with two webcams and a laser line projector," Proc. SPIE 9282, 7th International Symposium on Advanced Optical Manufacturing and Testing Technologies: Optical Test and Measurement Technology and Equipment, 92821T (18 September 2014) doi: 10.1117/12.2068094.

[18] C. Liu, S. Ma, Q. Ma, and X. Zeng, "Stereo vision system based on the laser dot matrix projection," Proc. SPIE 8197, 2011 International Conference on Optical Instruments and Technology: Optical Systems and Modern Optoelectronic Instruments, 819704 (17 November 2011); doi: 10.1117/12.906542.

[19] X. M. Xiong, C. J. Hua, C. J. Fang, Y. Chen, "Research on the 3D reconstruction method of the free-form surface based on the grid projection," Proc. SPIE 10033, Eighth International Conference on Digital Image Processing (ICDIP 2016), 100333B (29 August 2016); doi: 10.1117/12.2248319.

[20] S. Á. Guðmundsson, H. Aanæs, and R. Larsen, "Fusion Of Stereo Vision And Time-Of-Flight Imaging For Improved 3d Estimation," International Journal on Intelligent Systems Technologies and Applications (IJISTA), 2008.

[21] Y. M. Kim, C. Theobalt, J. Diebel, J. Kosecka, B. Miscusik, and S. Thrun, "Multi-View Image And Tof Sensor Fusion For Dense 3d Reconstruction," IEEE 12th International Conference on Computer Vision Workshops, ICCV Workshops, Kyoto, 2009.

[22] G. M. Um, K. Y. Kim, C. H. Ahn, and K. H. Lee, "Three-Dimensional Scene Reconstruction Using Multiview Images And Depth Camera," Proc. SPIE 5664, Stereoscopic Displays and Virtual Reality Systems XII, 2005.

[23] J. Peng, W. Xu, B. Liang and A. Wu, "Pose Measurement and Motion Estimation of Space NonCooperative Targets Based on Laser Radar and StereoVision Fusion," IEEE Sensors Journal, vol. 19, no. 8, 2019.

[24] K. Park, S. Kim and K. Sohn, "High-Precision Depth Estimation Using Uncalibrated LiDAR and Stereo Fusion," IEEE Transactions on Intelligent Transportation Systems, 2019.

[25] O. Yilmaz and F. Karakus, "Stereo And Kinect Fusion For Continuous 3D Reconstruction And Visual Odometry," International Conference on Electronics, Computer and Computation (ICECCO), Ankara, 2013.
[26] Y. Yemez, C. J. Wetherilt, “A Volumetric Fusion Technique For Surface Reconstruction From Silhouettes And Range Data," Computer Vision and Image Understanding, Elesvier, 2007.

[27] A. Pacheco, H. Bolívar, J. P. Espada, and R. G. Crespo, "Reconstruction Of High Resolution 3d Objects From Incomplete Images And 3d Information," International Journal of Interactive Multimedia and Artificial Intelligence, 2014.

[28] D. Maurer, Y. C. Ju, M. Breuß, A. Bruhn, "Combining Shape from Shading and Stereo: A Variational Approach for the Joint Estimation of Depth, Illumination and Albedo", In Proc. 27th British Machine Vision Conference, BMVC 2016, York, UK, September 2016 - R. Wilson, E. Hancock, W. Smith (Eds.), BMVA Press, Art. 76, 2016.

[29] E.Bylow, R. Maier, F. Kahl, and C. Olsson "Combining Depth Fusion and Photometric Stereo for Fine-Detailed 3D Models," (eds) Image Analysis. SCIA 2019. Lecture Notes in Computer Science, vol 11482. Springer, Cham, 2019.

[30] Aljo`sa O`sep, "Multi-View 3D Reconstruction of Highly-Specular Objects", Submitted to the Institute of Computer Science, Computer Graphics department, University of Bonn, Friedrich-EbertAllee 144, 53113 Bonn, march, 2013.

[31] Brian Curless, "3D Scanning, Acknowledgement: some content and figures", Slide Player, 2015.

[32] P. Corke, "Robotics, Vision and Control," Springer Tracts in Advanced Robotics Volume 118. 1st ed., Springer-Verlag Berlin Heidelberg, 2011.

[33] T. Moons, L. V. Gool, and M. Vergauwen, “3D Reconstruction from Multiple Images Part 1: Principles," Foundations and Trends in Computer Graphics and Vision, Vol. 4, No. 4 (2008) 287-398, 2009.

[34] R. Pisapia, "Disparity map extraction for a low cost 3D sensor", Master Thesis in Computer Engineering, Faculty of Engineering, 2015-16.

[35] S. Barone, P. Neri, A. Paoli, A. V. Razionale, "Flexible calibration of a stereo vision system by active display," Procedia Manufacturing, Volume 38, Elsevier, 2019.

[36] T. Li, C. Liu, Y. Liu, T. Wang, D. Yang, "Binocular stereo vision calibration based on alternate adjustment algorithm," Optik, Volume 173, Elsevier, 2018.

[37] J. Forest and Collado, "New Methods For Triangulation-Based Shape Acquisition Using Laser Scanners," PhD thesis, University of Girona, Department of Electronics, Information and Automation, Girona, 2004.

[38] B. Cyganek and J. P. Siebert, “An Introduction To 3D Computer Vision Techniques And Algorithms," Wiley, 1st edition, 2009.

[39] T. Ewbank, "Efficient and precise stereoscopic vision for humanoid robots", thesis submitted for the Master's degree in Computer Science Engineering, 
UNIVERSITY OF LIÈGE, Faculty of Applied Sciences, 2017.

[40] Morris and J. Anne, "Design of an Active Stereo Vision 3D Scene Reconstruction System Based on the Linear Position Sensor Module", Master's Thesis, University of Tennessee, Knoxville, 2006.

[41] X. Sun, Y. Jiang, Y. Ji, W. Fu, S. Yan, Q. Chen, B. Yu, and X. Gan, "Distance Measurement System Based on Binocular Stereo Vision," IOP Conference Series: Earth and Environmental Science, Volume 252, Issue 5, 2019.

[42] A. Zaarane, I. Slimani, W. Al Okaishi, I. Atouf, A. Hamdoun, "Distance measurement system for autonomous vehicles using stereo camera," Array, Volume 5, Elsevier, 2020.
[43] Y. Xue, T. Jia, W. Li, X. Yuan and D. Chen, "An improved method of depth measurement based on line laser," 2018 Chinese Control and Decision Conference (CCDC), Shenyang, IEEE, 2018.

[44] Y. Liu, H. Wang, and Q. Chen, "A Real-Time Car-Following Data Collecting Method Based on Binocular Stereo Vision," CICTP 2019: 19th COTA International Conference of Transportation Professionals in China-Connecting the World, 2019. [45] M. A. Isa, D. Sims-Waterhouse, S. Piano, and R. Leach, "Volumetric error modelling of a stereo vision system for error correction in photogrammetric threedimensional coordinate metrology", Precision Engineering, Elsevier, 2020. 